\title{
The whole picture: Holistic body posture recognition in infancy
}

\author{
Alyson Hock ${ }^{1} \cdot$ Hannah White ${ }^{1} \cdot$ Rachel Jubran $^{1} \cdot$ Ramesh S. Bhatt $^{1}$
}

Published online: 24 July 2015

(C) Psychonomic Society, Inc. 2015

\begin{abstract}
Holistic processing is tied to expertise and is characteristic of face and body perception by adults. Infants process faces holistically, but it is unknown whether they process body information holistically. In the present study, infants were tested for discrimination between body postures that differed in limb orientations in three conditions: in the context of the whole body, with just the isolated limbs that changed orientation, or with the limbs in the context of scrambled body parts. Five- and 9-month-olds discriminated between whole-body postures, but failed in the isolated-part and scrambled-body conditions, demonstrating holistic processing of information from bodies. These results indicate that at least some level of expertise in body processing develops quite early in life.
\end{abstract}

Keywords Social perception $\cdot$ Holistic processing $\cdot$ Body information processing $\cdot$ Infancy $\cdot$ Perceptual development

Adults can quickly and accurately detect social information from bodies, even in situations in which the face is not visible (de Gelder \& de Borst, 2015). This information can be used much like facial cues; for example, posture can be used to share approval, insult, intention, and emotion. Commonalities in the processing of faces and bodies have also been demonstrated. For instance, adults are thought to be experts on face processing because they represent faces holistically (McKone \& Robbins, 2011; Richler \& Gauthier, 2014; Tanaka \& Gordon, 2011). Similarly, the adult visual system applies a holistic strategy to

Ramesh S. Bhatt

rbhatt@email.uky.edu

1 Department of Psychology, University of Kentucky, Lexington, KY 40506-0044, USA process information from bodies (Reed, Stone, Grubb, \& McGoldrick, 2006; Robbins \& Coltheart, 2012; Seitz, 2002). In the present study, we examined the developmental origins of this specialized processing. Specifically, we examined whether 5- and 9-month-old infants process body posture holistically.

\section{Introduction}

\section{Holistic processing}

Holistic processing refers to a specific type of relational processing that results in images being represented as global entities rather than as disparate parts (Bartlett \& Searcy, 1993; Diamond \& Carey, 1986; Richler, Palmeri, \& Gauthier, 2012). Tanaka and Farah (1993) demonstrated the holistic nature of face processing using a feature recognition task (e.g., "identify Jim's nose"). Recognition was measured under two conditions: In one, the parts were presented in the context of the whole face; in the other, the parts were presented in isolation. Participants were less accurate in the isolated-part than in the whole-face condition, indicating that faces were processed holistically. Thus, the Tanaka and Farah (1993) part-whole task examines discrimination between parts individually and in the context of the whole face, and superior performance in the latter condition is taken to imply holistic processing (Tanaka \& Farah, 2003; Tanaka \& Gordon, 2011).

Seitz (2002) extended the part-whole paradigm to body processing in adults and children. She found that 8- and 10year-old children, as well as adults, recognized body parts more accurately when parts were presented in the context of the whole body than when they were presented in isolation. Reed and colleagues (2006) tested adults' recognition of whole bodies, isolated parts, and scrambled bodies. Adults exhibited an inversion effect with whole bodies, but not with 
parts or scrambled images. These results are consistent with the part-whole difference found by Tanaka and Farah (1993) and by Seitz (2002; see also Robbins \& Coltheart, 2012; Willems, Vrancken, Germeys, \& Verfaillie, 2014). Thus, body processing is holistic in adulthood and childhood. However, it is unknown whether infants also process bodies holistically. We addressed this issue in the present study.

\section{Development of body processing in infancy}

There is considerable evidence of the rapid development of face processing expertise early in life (Lee, Anzures, Quinn, Pascalis, \& Slater, 2011). However, not much is known about body processing. A few recent studies have suggested that infants have some knowledge about the structure and organization of human bodies. Zieber, Kangas, Hock, and Bhatt (2015) found that 3.5-month-old infants discriminate between proportional and distorted bodies and between typical and scrambled bodies (see also Zieber et al., 2010). Additionally, 6.5-month-olds match static body postures to emotions, indicating infants' sensitivity to gestures (Zieber, Kangas, Hock, \& Bhatt, 2014). Heron-Delaney, Quinn, Lee, Slater, and Pascalis (2013) found that 9-month-olds had a preference for unattractive male bodies over attractive ones.

However, prior research by Slaughter and colleagues (for a review, see Slaughter, Heron-Delaney, \& Christie, 2012) suggested that body knowledge in infancy is not robust until the second year of life. Moreover, the discrimination between intact and scrambled/distorted bodies found by Zieber and colleagues (Zieber et al., 2010; Zieber et al., 2015) is not necessarily indicative of holistic processing. This is because, although infants may be sensitive to the relative size and location of body parts, they may still represent features individually and not process the body image as an integrated whole. Consistent with this logic, previous research with faces suggested that first-order relation processing and holistic processing are distinct and separable processes (Maurer, Le Grand, \& Mondloch, 2002). Thus, the question of whether infants process body information holistically has not been addressed previously.

In the present study, we examined whether 5- and 9-montholds discriminate postural differences induced by changes in limb positions better in the context of the whole body than in isolation. If so, it would indicate holistic processing (Seitz, 2002; Tanaka \& Farah, 1993). However, this result might not necessarily indicate specific knowledge about bodies. Infants may be able to detect orientation changes as long as a sufficient context is provided, even if this context is not the typical body. In other words, infants' detection of limb orientation changes in the whole-body condition might not be based on their knowledge about bodies per se, but rather on the presence of some context that anchors the limbs.

To address this issue, an additional group of infants was tested with scrambled bodies in which all parts of the normal whole body were present, albeit in a reorganized fashion (Fig. 1). Scrambling preserves the low-level features of normal bodies, such as contrast, and distorts only the configural properties - that is, the unique overall organization used to signify a human body as opposed to another object. If, despite the presence of all parts, infants fail to discriminate the same changes that they discriminated in the whole-body condition, this would suggest that the holistic processing is a reflection of body-specific processing (Reed et al., 2006; Tanaka \& Farah, 1993). Thus, three groups of 5 -month-olds and three groups of 9-month-olds were tested, one with the whole body, another with just the isolated parts, and the third with scrambled body parts.

We chose to study 5-month-olds because prior research had provided clear evidence of configural information processing of faces at 5 months of age (Bhatt, Bertin, Hayden, \& Reed, 2005; Hayden, Bhatt, Reed, Corbly, \& Joseph, 2007). We also tested an older age group ( 9 months) because some researchers have claimed that body knowledge is slower to develop than face knowledge (Slaughter et al., 2012), and it is possible that holistic body information processing might be evident only by this age. Moreover, we examined posture discrimination because posture signals a variety of socially significant information, such as emotion, intention, and forthcoming actions (Dael, Mortillaro, \& Scherer, 2012; de Gelder, 2006; Tomasello, Carpenter, Call, Behne, \& Moll, 2005). Therefore, the ability to efficiently discriminate between various body postures is critical for social functioning.

\section{Method}

\section{Participants}

The participants were 54 five-month-old infants (mean age $=$ 150.98 days, $S D=6.47 ; 32$ male, 22 female) and 36 ninemonth-old infants (mean age $=274.33$ days, $S D=8.93 ; 17$ male, 19 female). We chose to use larger sample sizes at 5 months than at 9 months because younger infants typically exhibit more variability in their performance, and a power analysis conducted using data from prior studies from our lab showed that these sample sizes would be sufficient to detect an effect size $d$ of 0.60 in $t$ tests with a power of .80 .

Infants were recruited through birth announcements and a local hospital. They were predominately Caucasian and from middle-class families. The data from two additional 5-monthold infants and an additional 9-month-old infant were excluded due to side bias.

\section{Stimuli}

The stimuli, created using the Poser 2.0 software (Curious Labs, Santa Cruz, CA), were female figures whose arms and 


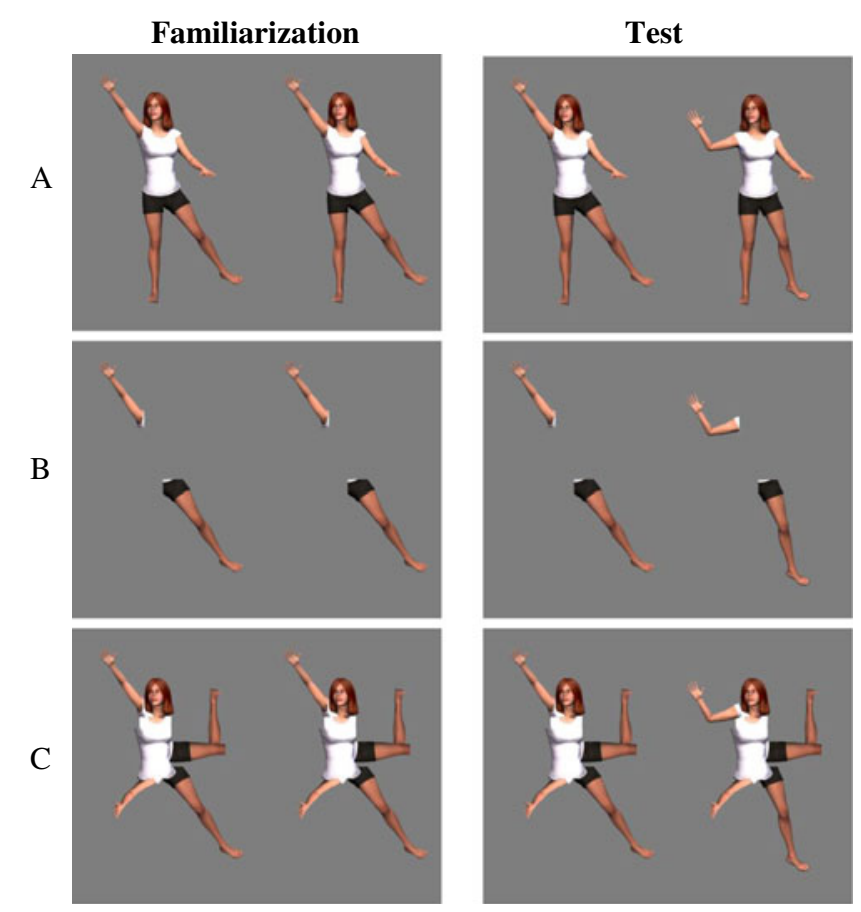

Fig. 1 Examples of the whole-body (a), isolated-body-part (b), and scrambled (c) familiarization and test stimuli. In each condition, infants were familiarized to an image containing two identical body postures and then tested with the familiarization posture paired with a novel body posture. The novel postures were created by changing the orientations of an arm and a leg.

legs were positioned in different orientations to create novel poses (Fig. 1). Six upright physically possible body postures were constructed using three different bodies, with two body posture variations for each body. The second variation of each figure was constructed by altering the orientation of one arm and one leg of the figure (Fig. 1). The degrees of the changes in orientation of the arm and leg were approximately the same across the three pairs.

Isolated-body-part stimuli were created from the wholebody stimuli. Recall that the whole-body posture changes involved only an arm and a leg. The isolated-part stimuli were created by presenting only these parts and omitting the rest of the body (Fig. 1). The isolated arm and leg parts remained in the exact locations they had occupied in the whole-body condition. In other words, the pose changes depicted in the whole-body stimuli were exactly duplicated in the body-part stimuli, except that only the parts that were involved in the pose changes were visible, and the rest of the body was not presented (Fig. 1).

Scrambled-body stimuli were also created from the wholebody stimuli. The critical arm and leg whose orientations were changed in each stimulus were, once again, left in the same position; however, the remaining body parts (torso, arm, and leg) were moved to new locations and reattached in novel configurations (Fig. 1). The position of the head was not altered, so that any effects of scrambling could be attributable to body part reorganization rather than to the displacement of the head.

Female bodies were used in this study because research suggests that infants exhibit a preference for females over males and also exhibit a greater degree of expertise on female stimuli (e.g., Quinn, Yahr, Kuhn, Slater, \& Pascalis, 2002; Ramsey, Langlois, \& Marti, 2005). Therefore, female bodies could potentially induce infants to display greater knowledge about human bodies.

\section{Apparatus}

Infants were seated on their parent's lap approximately $45 \mathrm{~cm}$ in front of a $50-\mathrm{cm}$ computer monitor in a darkened chamber. The parents wore opaque darkened glasses that prevented them from viewing the stimuli. A video camera located on top of the monitor was used to monitor and record the infants' performance for later offline coding.

\section{Procedure}

We utilized a familiarization-novelty preference procedure that has been used in several previous studies (e.g., Pascalis, de Haan, \& Nelson, 2002; Scott \& Monesson, 2009; Zieber et al., 2015). Each familiarization and test trial was preceded by an attention-getter in which alternating green and purple shapes appeared in the center of the monitor. During familiarization, the infants were simultaneously exposed to two identical copies of a body posture. The bodies remained on the screen until the infant had accumulated $30 \mathrm{~s}$ of look duration to the stimuli. Immediately following the single familiarization trial, infants were tested on two 8-s test trials in which the familiar body posture was presented on one side while the same body, in a novel posture, was presented on the other side. Infants were tested for their preference between the familiar and novel body postures. In studies using this kind of procedure, infants tend to look longer at the novel image (Pascalis et al., 2002).

The left-right position of the novel body posture during the first test trial was counterbalanced across participants and reversed during the second test trial. A third of the infants at each age were tested on one of the three body pairs. In addition, the familiarization and test stimuli were counterbalanced within each age, so that both postures for each body served equally often as the familiarization and novel test stimuli. That is, half of the infants were familiarized to a body pose and tested with the same body depicting a second pose as the novel stimulus, whereas the other half of the infants were familiarized to the second pose and tested with the first pose as the novel stimulus.

Video coding was completed offline by a coder blinded to the experimental condition and the left-right locations of the stimuli. The video was played back at $25 \%$ of the normal 
speed during coding. The data from $25 \%$ of the infants were coded by a second observer to establish reliability. The intraclass correlation coefficients (ICC) were .98 for the 5month-olds and .97 for the 9-month-olds. Typically, ICC values greater than .9 are interpreted as indicating excellent agreement between raters.

As in prior studies (e.g., Bhatt et al., 2005; Scott \& Monesson, 2009), the dependent measure was the percent preference for the novel body posture across the two test trials. This was calculated by dividing the total looking time toward the novel body posture across the two trials by the total looking time toward both the novel and familiar body postures across the two trials, and multiplying this ratio by 100 .

\section{Results}

The mean times required for infants to accumulate $30 \mathrm{~s}$ of looking during familiarization are presented in Table 1. An Age (5 months, 9 months) $\times$ Condition (whole body, part, scrambled) analysis of variance (ANOVA) failed to reveal significant main effects [age: $F(1,84)=0.47, p=.49, \eta_{\mathrm{p}}{ }^{2}=$ .006 ; condition: $\left.F(2,84)=0.43, p=.65, \eta_{\mathrm{p}}{ }^{2}=.01\right]$ or an interaction $\left[F(2,84)=0.57, p=.57, \eta_{\mathrm{p}}{ }^{2}=.013\right]$. Thus, we found no evidence to suggest differences in the patterns of familiarization to the three kinds of stimuli or between age groups.

Infants' mean novelty preference scores during the test trials are also shown in Table 1. Both 5- and 9-month-olds exhibited scores that were significantly greater than the chance level of $50 \%$ in the whole-body condition [5-month-olds: $t(17)=2.84, p=.01$, two-tailed, $d=1.38$; 9-month-olds: $t(11)=5.94, p=.001$, two-tailed, $d=3.58$ ] but not in the part or scrambled conditions (all $p \mathrm{~s}>.60$ ). Thus, at both ages, infants discriminated posture changes only in the context of the intact whole body.

Moreover, an Age (5 months, 9 months) $\times$ Condition (whole body, part, scrambled) ANOVA on infants' mean novelty scores indicated a significant main effect of condition, $F(2,84)=3.23, p=.04, \eta_{\mathrm{p}}{ }^{2}=.07$, but neither the interaction between age and condition $\left[F(2,84)=0.02, p=.99, \eta_{\mathrm{p}}{ }^{2}=\right.$ $.001]$ nor the main effect of age $\left[F(1,84)=0.12, p=.73, \eta_{\mathrm{p}}{ }^{2}=\right.$ $.001]$ was significant. Given that Age was not a significant factor, we collapsed across ages in the subsequent analyses. Planned $t$ tests revealed that the mean novelty preference score in the whole-body condition was significantly greater than the score in the part $[t(58)=2.11, p=.039$, two-tailed, $d=0.55]$ and the scrambled $[t(58)=2.65, p=.01$, two-tailed, $d=0.68]$ conditions. These results indicate that 5-and 9-month-olds discriminated posture changes involving limb orientations only in the context of the intact human body, thereby indicating holistic processing.

\section{Discussion}

Five- and 9-month-old infants discriminated changes in the orientation of limbs within the context of a typical whole body, but failed to detect the same changes when body parts were presented in isolation or in the context of scrambled bodies. These findings indicate that, like adults, infants process body information holistically. To our knowledge, this is the first study to have demonstrated this kind of expert body information processing in infancy.

Holistic processing presumably enables infants and adults to process information efficiently and effectively. The fact that infants as young as 5 months of age also process body information holistically thus suggests that at least some level of expertise for body information is available by the first half of the first year of life. It is possible that infants have enough experience with bodies by 5 months of age to develop this level of expertise. Alternatively, experience by itself may not be sufficient to lead to holistic processing, but experience in conjunction with biological "preparedness" might enable infants this age to attain this level of sophisticated processing. That is, some innate basic representation of bodies available at

Table 1 Time to accumulate $30 \mathrm{~s}$ of exposure during familiarization, look durations to novel and familiar postures, and percent preference for the novel stimulus during test trials (standard errors are in parentheses)

\begin{tabular}{llllllll}
\hline & Condition & $N$ & $\begin{array}{l}\text { Mean Time to Accumulate } \\
\text { 30 s Looking }\end{array}$ & $\begin{array}{l}\text { Mean Look Duration } \\
\text { to Novel Posture }\end{array}$ & $\begin{array}{l}\text { Mean Look Duration } \\
\text { to Familiar Posture }\end{array}$ & $\begin{array}{l}\text { Mean Novelty } \\
\text { Preference (\%) }\end{array}$ \\
\hline 9-month-olds (vs. 50 \% Chance) \\
\end{tabular}

${ }^{*} p<.02,{ }^{* *} p<.001$, two-tailed; both indicate results significantly different from chance $(50 \%)$. 
birth, combined with postnatal experience, might lead to the rapid development of holistic processing mechanisms that enable infants to process body information in a sophisticated manner. The latter account is consistent with many models of face processing that assume that innate proclivities to attend to faces or face-like images at birth, combined with extensive postnatal experience, lead to specialized processing of faces in infancy (Johnson, 2011; Lee et al., 2011).

The evidence of body processing expertise found in the present study is consistent with prior research suggesting that infants during the first year of life are sensitive to many structural aspects of bodies, such as the arrangement and relative sizes of the body parts (Gliga \& Dehaene-Lambertz, 2005; Zieber et al., 2015), the relation between sex and body shape (Hock, Kangas, Zieber, \& Bhatt, 2015), and the relative attractiveness of body shapes (Heron-Delaney et al., 2013). However, the present findings are not consistent with the claim by Slaughter and her colleagues (Slaughter \& Heron, 2004; Slaughter et al., 2012) that body knowledge is quite limited until the second year of life. It is not clear why the Slaughter studies led to different conclusions. Many of the experiments by Slaughter and colleagues involved the examination of infants' sensitivity to intact versus reorganized images, whereas the present research examined infants' perception of posture, and it is possible that posture perception relies more on a holistic processing system (but see Gliga \& DehaeneLambertz, 2005, and Zieber et al., 2015, for evidence indicating that young infants are sensitive to body feature reorganization). Also, in the present experiment we used female body images, whereas many of the Slaughter studies utilized male images. It is known that infants perform better on female than on male images in a variety of discrimination and categorization tasks (if their primary caregivers are female; see Quinn et al., 2002; Ramsey et al., 2005). Consequently, the female images used in the present study may have facilitated performance. It is also possible that the paired-comparison procedure used in the present study (in which novel and familiar stimuli were presented side by side) was more sensitive than the successive-comparison procedures typically used by Slaughter and her colleagues (in which only one stimulus is presented at a time, so that novel and familiar stimuli have to be compared sequentially). Thus, some combination of stimulus and procedural differences between the present study and the Slaughter studies likely led to the different conclusions.

Another point worth noting concerns the relative rates of development of holistic body and face processing. Turati, Di Giorgio, Bardi, and Simion (2010) found that 3-month-olds, but not newborns, process faces holistically. Using a different procedure, Cashon and Cohen (2004) found that 4-montholds, but not 3-month-olds, process faces holistically; however, even some older infants, such as 6.25-month-olds, failed to exhibit evidence of holistic processing under the same circumstances. Also, Bhatt et al. (2005) found that 5-montholds discriminate subtle spatial relations in faces (such as the distance between the eyes) that are thought to underlie holistic image processing, but 3.5-month-olds do not. Thus, it appears that holistic face processing might be available anytime from 3 to 7 months of age. The fact that the 5-month-olds in the present experiment exhibited evidence of holistic body processing thus suggests that there is unlikely to be a large gap in the development of the holistic processing mechanisms underlying face versus body processing. This indicates the rapid development of both face and body information processing early in life, which presumably allows the developing infant to efficiently process important social information.

Author note This research was supported by grants from the National Science Foundation (No. BCS-1121096) and the National Institute of Child Health and Human Development (No. HD075829). The authors thank the infants and parents who participated in this study.

\section{References}

Bartlett, J. C., \& Searcy, J. (1993). Inversion and configuration of faces. Cognitive Psychology, 25, 281-316.

Bhatt, R. S., Bertin, E., Hayden, A., \& Reed, A. (2005). Face processing in infancy: Developmental changes in the use of different kinds of relational information. Child Development, 76, 169-181.

Cashon, C. H., \& Cohen, L. B. (2004). Beyond U-shaped development in infants' processing of faces: An information-processing account. Journal of Cognition and Development, 5, 59-80.

Dael, N., Mortillaro, M., \& Scherer, K. R. (2012). Emotion expression in body action and posture. Emotion, 12, 1085-1101. doi:10.1037/ a0025737

de Gelder, B. (2006). Towards the neurobiology of emotional body language. Nature Reviews Neuroscience, 7, 242-249.

de Gelder, B., \& de Borst, A. W. (2015). Body perception. In A. W. Toga (Ed.), Brain mapping: An encyclopedic reference (Vol. 3, pp. 107114). London, UK: Elsevier Academic Press. doi:10.1016/B978-012-397025-1.00162-7

Diamond, R., \& Carey, S. (1986). Why faces are and are not special: An effect of expertise. Journal of Experimental Psychology: General, 115, 107-117. doi:10.1037/0096-3445.115.2.107

Gliga, T., \& Dehaene-Lambertz, G. (2005). Structural encoding of body and face in human infants and adults. Journal of Cognitive Neuroscience, 17, 1328-1340.

Hayden, A., Bhatt, R. S., Reed, A., Corbly, C. R., \& Joseph, J. E. (2007). The development of expert face processing: Are infants sensitive to normal differences in second-order relational information? Journal of Experimental Child Psychology, 97, 85-98.

Heron-Delaney, M., Quinn, P. C., Lee, K., Slater, A. M., \& Pascalis, O. (2013). Nine-month-old infants prefer unattractive bodies over attractive bodies. Journal of Experimental Child Psychology, 115, 3041. doi:10.1016/j.jecp.2012.12.008

Hock, A., Kangas, A., Zieber, N., \& Bhatt, R. S. (2015). The development of sex category representation in infancy: Matching of faces and bodies. Developmental Psychology, 51, 346-352.

Johnson, M. H. (2011). Face perception: A developmental perspective. In A. J. Calder, G. Rhodes, M. H. Johnson, \& J. V. Haxby (Eds.), The Oxford handbook of face perception (pp. 3-14). Oxford, UK: Oxford University Press. doi:10.1093/oxfordhb/9780199559053. 013.0001 
Lee, K., Anzures, G., Quinn, P. C., Pascalis, O., \& Slater, A. (2011). Development of face processing expertise. In A. J. Calder, G. Rhodes, M. H. Johnson, \& J. V. Haxby (Eds.), The Oxford handbook of face perception (pp. 753-778). Oxford, UK: Oxford University Press.

Maurer, D., Le Grand, R., \& Mondloch, C. J. (2002). The many faces of configural processing. Trends in Cognitive Sciences, 6, 255-260.

McKone, E., \& Robbins, R. (2011). Are faces special? In A. J. Calder, G. Rhodes, M. H. Johnson, \& J. V. Haxby (Eds.), The Oxford handbook of face perception (pp. 149-176). Oxford, UK: Oxford University Press. doi:10.1093/oxfordhb/9780199559053.013.0009

Pascalis, O., de Haan, M., \& Nelson, C. A. (2002). Is face processing species-specific during the first year of life? Science, 296, 1321-1323.

Quinn, P. C., Yahr, J., Kuhn, A., Slater, A. M., \& Pascalis, O. (2002). Representation of the gender of human faces by infants: A preference for female. Perception, 31, 1109-1122.

Ramsey, J. L., Langlois, J. H., \& Marti, N. C. (2005). Infant categorization of faces: Ladies first. Developmental Review, 25, 212-246.

Reed, C. L., Stone, V. E., Grubb, J. D., \& McGoldrick, J. E. (2006). Turning configural processing upside down: Part and whole body postures. Journal of Experimental Psychology: Human Perception and Performance, 32, 73-87. doi:10.1037/0096-1523.32.1.73

Richler, J. J., \& Gauthier, I. (2014). A meta-analysis and review of holistic face processing. Psychological Bulletin, 140, 1281-1302.

Richler, J. J., Palmeri, T. J., \& Gauthier, I. (2012). Meanings, mechanisms, and measures of holistic processing. Frontiers in Psychology, 3, 553. doi:10.3389/fpsyg.2012.00553

Robbins, R. A., \& Coltheart, M. (2012). The effects of inversion and familiarity on face versus body cues to person recognition. Journal of Experimental Psychology: Human Perception and Performance, 38, 1098-1104.

Scott, L. S., \& Monesson, A. (2009). The origin of biases in face perception. Psychological Science, 20, 676-680.

Seitz, K. (2002). Parts and wholes in person recognition: Developmental trends. Journal of Experimental Child Psychology, 82, 367-381.

Slaughter, V., \& Heron, M. (2004). Origins and early development of human body knowledge. Monographs of the Society for Research in Child Development, 69(2), 1-102.
Slaughter, V., Heron-Delaney, M., \& Christie, T. (2012). Developing expertise in human body perception. In V. Slaughter \& C. Brownell (Eds.), Early development of body representations (pp. 81-100). Cambridge: Cambridge University Press.

Tanaka, J. W., \& Farah, M. J. (1993). Parts and wholes in face recognition. Quarterly Journal of Experimental Psychology, 46A, 225-245. doi:10.1080/14640749308401045

Tanaka, J. W., \& Farah, M. J. (2003). The holistic representation of faces. In M. A. Peterson \& G. Rhodes (Eds.), Perception of faces, objects, and scenes: Analytic and holistic processes (pp. 53-74). Oxford, UK: Oxford University Press. doi:10.1093/acprof:oso/ 9780195313659.003.0003

Tanaka, J. W., \& Gordon, I. (2011). Features, configuration, and holistic face processing. In A. J. Calder, G. Rhodes, M. H. Johnson, \& J. V. Haxby (Eds.), The Oxford handbook of face perception (pp. 177194). Oxford, UK: Oxford University Press. doi:10.1093/oxfordhb/ 9780199559053.013.0010

Tomasello, M., Carpenter, M., Call, J., Behne, T., \& Moll, H. (2005). Understanding and sharing intentions: The origins of cultural cognition. Behavioral and Brain Sciences, 28, 675-691. doi:10.1017/ S0140525X05000129. disc. 691-735.

Turati, C., Di Giorgio, E., Bardi, L., \& Simion, F. (2010). Holistic face processing in newborns, 3-month-old infants, and adults: Evidence from the composite face effect. Child Development, 81, 1894-1905.

Willems, S., Vrancken, L., Germeys, F., \& Verfaillie, K. (2014). Holistic processing of human body postures: Evidence from the composite effect. Frontiers in Psychology, 5, 618. doi:10.3389/fpsyg.2014. 00618

Zieber, N., Bhatt, R. S., Hayden, A., Kangas, A., Collins, R., \& Bada, H. (2010). Body representation in the first year of life. Infancy, 15, 534 544.

Zieber, N., Kangas, A., Hock, A., \& Bhatt, R. S. (2014). The development of intermodal emotion perception from bodies and voices. Journal of Experimental Child Psychology, 126, 68-79.

Zieber, N., Kangas, A., Hock, A., \& Bhatt, R. S. (2015). Body structure perception in infancy. Infancy, 20, 1-17. 